\title{
Pulmonary hamartoma, a rare benign tumour of the lung - Case series
}

\author{
Tyagi Ruchita', Bal Amajit', Mahajan Divyesh², Nijhawan Raje ${ }^{3}$, Das Ashim ${ }^{4}$ \\ ${ }^{1}$ Department of Pathology, PGIMER, Chandigarh, ${ }^{2}$ Department of Radiodiagnosis, PGIMER, Chandigarh, ${ }^{3}$ Department of Cytology and \\ Gynecologic Pathology, PGIMER, Chandigarh, ${ }^{4}$ Department of Histopathology, PGIMER, Chandigarh
}

Introduction: Pulmonary hamartoma, with incidence of $0.25-0.32 \%$, accounts for $6 \%$ of solitary pulmonary nodules. The role of radiology is limited as only $10-30 \%$ of cases show characteristic 'popcorn' calcification and Computed Tomography can detect approximately $50 \%$ of hamartomas. Hence cytological and/or histopathological examination is required to make a definitive diagnosis and exclude malignancy. Objective: As pulmonary hamartoma is a rare entity detected serendipitously on radiography and requires cytological and histopathological examination for confirmation of diagnosis, we present nine cases of solitary pulmonary nodules which were diagnosed as pulmonary hamartoma. Methods: We retrospectively screened departmental records and slides and found nine cases of pulmonary hamartoma in our tertiary care institute (Post Graduate Institute of Medical Education and Research, Chandigarh, India). Three cases were diagnosed on CT guided Fine Needle Aspiration Cytology and four cases were diagnosed on histopathological examination ofsurgical specimens, over a period of 16 years (1997-2012). Two cases were incidentally discovered to have pulmonary hamartoma at autopsy. Observations: The age of the patients ranged from 17-63 years (mean-46.3), with male to female ratio being 3.5:1. The size of the hamartoma varied from $0.4-1.3 \mathrm{~cm}$, with mean diameter of $1 \mathrm{~cm}$. Cytology showed mixture of bronchial epithelial cells, adipocytes and stromal fragments in fibromyxoid and chondroid background. Histopathology demonstrated lobules of cartilage and adipose tissue with intervening clefts lined by respiratory epithelium and mesenchymal stroma. Conclusion: Every solitary pulmonary nodule is not malignant. It is important to correctly diagnose pulmonary hamartoma, a rare, yet benign neoplasm presenting as a solitary lung nodule and distinguish it from malignancy.

Key words: Hamartoma, Nodule, Pulmonary, Solitary

\section{INTRODUCTION}

The term " hamartoma" from a Greek word meaning 'to err' was coined by Albrecht (1904), who defined it as "a tumour-like malformation in which one can demonstrate only an abnormal mixture of the normal developmental components of the organ in which they occur, whether it be with regard to the quantity, to the arrangement, or to the degree of development, or in all three respects". ${ }^{1}$ However, a recent hypothesis suggests a neoplastic origin of pulmonary hamartomas, instead of considering them as developmental malformations. ${ }^{2}$

With incidence of $0.25-0.32 \%$, pulmonary hamartoma is the most common benign lesion of the lung, and accounts for $6 \%$ of solitary pulmonary nodules which may also be caused by malignancy, metastasis, granuloma or other benign tumours. ${ }^{3-5}$ Most of these lesions are asymptomatic, except when they are endobronchial in location and cause obstruction, and are detected serendipitously on radiology as solitary pulmonary nodule. These lesions have heterogeneous appearance on radiology, thus radiology has a limited role in distinguishing pulmonary hamartoma from other causes of solitary pulmonary nodule which include malignancy, metastasis, granuloma or other benign neoplasms. The characteristic "popcorn" calcification is seen in only $10-30 \%$ of cases and CT can detect about $50 \%$ of hamartomas. ${ }^{6,7}$ Therefore, cytological and/or histopathological examination is required for a definitive diagnosis or at least to exclude malignancy. 


\section{MATERIAL AND METHODS}

A retrospective analysis of Departmental slides and records of Post Graduate Institute of Medical Education and Research, Chandigarh (India) - a tertiary care centre, revealed nine cases of pulmonary hamartoma in our tertiary care institute, over a period of 16 years (January 1996-December 2012). In two cases, pulmonary hamartoma was an incidental finding on autopsy. Three cases underwent CT guided Fine Needle Aspiration Cytology using 22 gauge needle and the aspiration smears, stained with May Grunwald Giemsa and hematoxylin and eosin, were studied. The excision of the lung nodules was done in four cases and the specimens were sent for histopathological examination. Formalin fixed paraffin embedded tissue sections stained with hematoxylin and eosin were subjected to light microscopic examination.

\section{OBSERVATIONS}

\section{Clinical history}

The age of our patients varied from 17-63 years, with mean age being 49 years. There was a male preponderance as the male to female ratio was 3.5:1. Three patients were asymptomatic and were incidentally detected to have solitary pulmonary nodules in upper lobe of left lung, and in lower and upper lobe of right lung respectively, on radiology done for other purposes. One of the cases, a known case of squamous cell carcinoma of tongue, has been reported recently, and was found to have a solitary nodule in the upper lobe of right lung which was suspicious of either a metastatic deposit or a synchronous primary malignancy of lung ${ }^{8}$ (Figure 1).

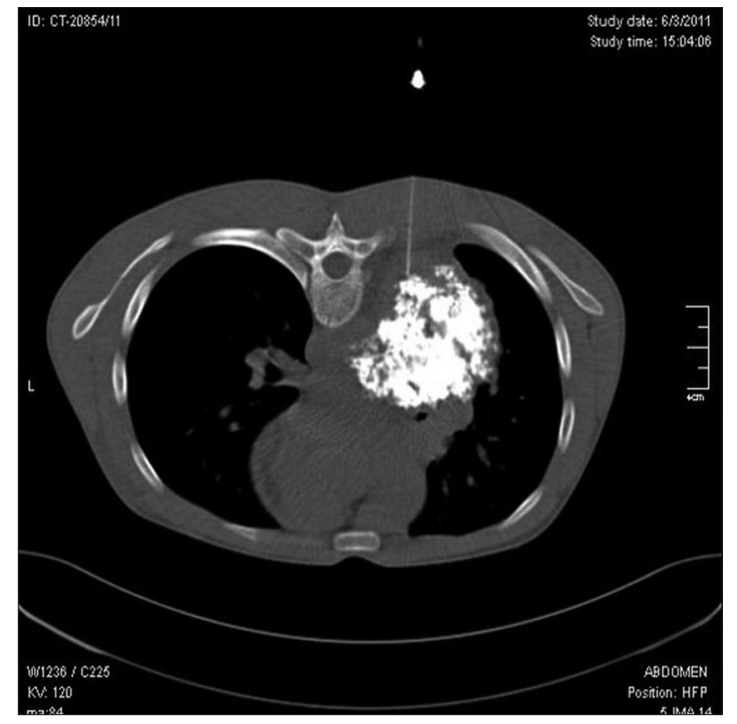

Figure 1: CT guided FNAC of solitary pulmonary nodule with characteristic popcorn calcification in parenchyma of right lung
The patient with endobronchial hamartoma presented with recurrent pneumonia, while two cases with solitary pulmonary nodules were suspected to have carcinoma lung. One of the autopsy cases had been operated previously for right sided temporo-parietal astrocytoma. Both the lungs had fibrocaseous lesions at the apex. The middle lobe of right lung, in addition, showed a $1.3 \mathrm{~cm}$ diameter nodule which turned out to be pulmonary hamartoma on histopathological examination. The other autopsy case was a case of small cell carcinoma which had spread to hilar, paratracheal and mediastinal lymph nodes as well as to the liver. The lower lobe of the right lung showed a whitish firm nodule which was shown to be hamartoma on microscopy.

\section{Cytology findings}

The smears were of poor cellularity but showed chondromyxoid stroma in the background. There were few bronchial epithelial cells scattered in the smears. The classical cytological features seen were epithelial and spindle shaped cells intermingled with adipocytes in a background of chondroid material. However, in one case epithelial cells were few and the predominant component was mesenchymal, represented by spindle shaped cells embedded in fibromyxoid material. One case in addition showed occasional foci of calcification (Figure 2). The epithelial cells did not show any reactive nuclear atypia.

\section{Histopathology findings}

A total of six cases were studied histologically. Grossly, the excised specimens comprised of firm to hard nodules of $0.2-1.3 \mathrm{~cm}$, with a mean size of $1 \mathrm{~cm}$. Cut section showed greyish white, glistening lobules of cartilage. Microscopically, these were well circumscribed, un-encapsulated lesions comprising of lobules of mature cartilage and separated by

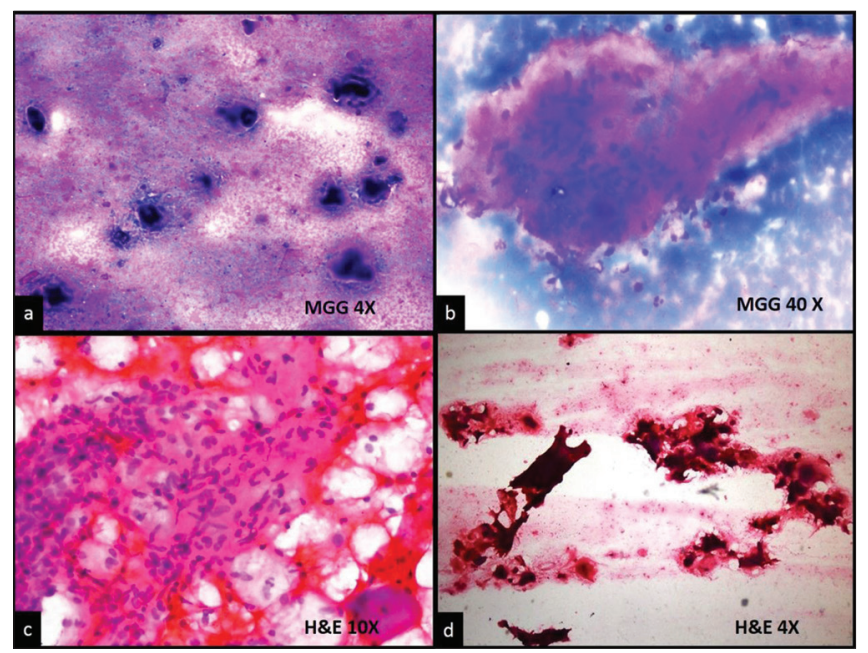

Figure 2: FNAC smears showing (a) chondroid stromal material in the background; (b) spindle cells in chondroid stromal material; (c) clusters of spindle cells and fragments of cartilage; (d) occasional foci of calcification in chondroid background 
intervening clefts lined by cuboidal to columnar respiratory epithelium which was forming glands at places. The adipose tissue, fibroconnective stroma, occasional bundles of smooth muscle cells and foci of calcification were seen admixed with cartilage (Figure 3). The surrounding lung parenchyma was unremarkable or showed deposition of anthracotic pigment. However, no anthracotic pigment deposition was seen within the lesion.

\section{DISCUSSION}

Pulmonary hamartomas are rare tumours, with incidence of $0.25-0.32 \%$ in general population. ${ }^{3}$ The peak incidence reported is between the sixth and seventh decades, with a $2: 1$ or $3: 1$ male preponderance. ${ }^{7,9}$ In our study, the age of the cases ranged from 17-63 years (mean - 49 years) with male to female ratio being 3.5:1.

On radiology, a rounded shadow with well-defined margins and surrounded by normal looking lung parenchyma, with occasional foci of calcification in the shadow, in an asymptomatic person; strongly suggests the possibility of a hamartoma. However, the typical 'popcorn' calcification is present in only $10-30 \%$ of chest radiographs. CT scans are more sensitive for detecting calcification and adipose tissue in about $50 \%$ of hamartomas but they may not be able to demonstrate the salient features each time and it may be difficult to distinguish benign from malignant lesions. ${ }^{6,7}$

Flexible fibre optic bronchoscopic transbronchial biopsy has been suggested as a first line technique to diagnose endobronchial hamartomas. The peripherally situated parenchymal hamartomas can be diagnosed

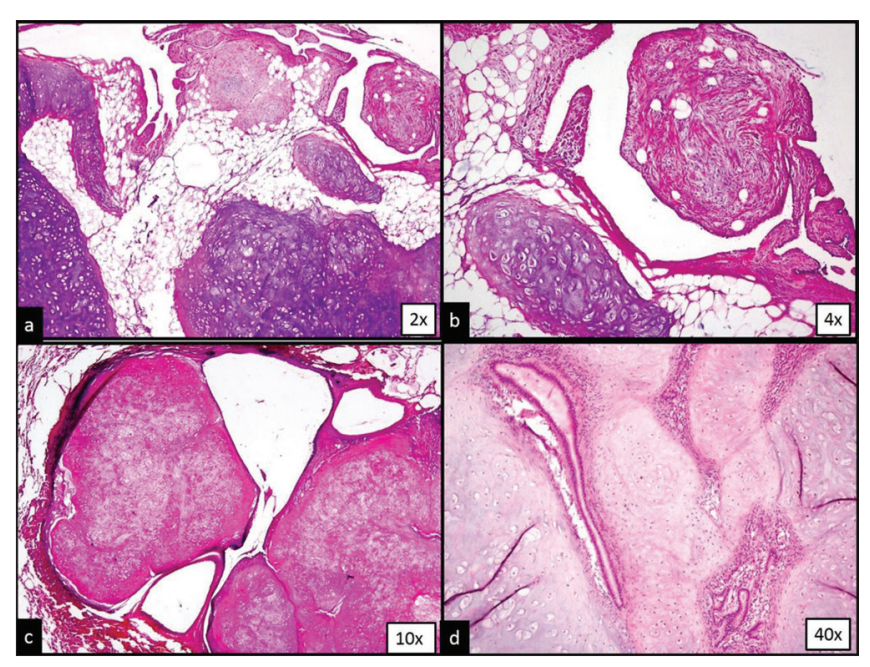

Figure 3: Photomicrographs ofhistopathology showing hyaline cartilage, adipose tissue, fibroconnective stroma and smooth muscle bundles, lobules of mature cartilage with intervening clefts lined by cuboidal to columnar respiratory epithelium, forming glands focally (d) high power view of clefts lined by respiratory epithelium. by transthoracic FNAC or wedge resection. ${ }^{7}$ Epithelial component may be represented by sheets of small, uniform, cuboidal or columnar bronchial epithelial cells and bare nuclei. Mesenchymal component may be subtle and may be represented by tissue such as fat, smooth muscle, cartilage, fibrous tissue and occasionally bone. Fibromyxoid stroma, seen as stromal material intermixed with spindled cells and chondroid material seen as dense, homogenous glassy or slightly refractile material with or without lacunae helps to easily identify these lesions on cytology. ${ }^{9}$ The aspirate smears of pulmonary hamartoma may be poorly cellular due to the dense nature of the lesion or they may be highly cellular with cells having granular chromatin, mimicking a neuroendocrine tumour. Hughes et al showed $78 \%$ specificity and $22 \%$ false-positive rate in FNA diagnosis. Most common false-positive diagnoses were carcinoid tumor, adenocarcinoma and small cell carcinoma. Paucity of stroma and prominent epithelial component led to high false-positive rate in their study. ${ }^{10}$ The fibromyxoid background may be mistaken for mucin. Hence, pulmonary hamartomas may be misdiagnosed as carcinoid tumours, adenocarcinoma, small cell carcinoma, adenoid cystic carcinoma, mucoepidermoid carcinoma, granular cell tumour, bronchogenic carcinoma, sarcomatoid carcinoma or infectious granulomata on cytology. Careful examination of smears to demonstrate bronchial epithelial cells intermingled with adipocytes in chondroid matrix helps to correctly identify this lesion. ${ }^{11}$

The majority of the hamartomas are intraparenchymal while endobronchial hamartomas comprise 10-20\% of hamartomas., ${ }^{3,12}$ In our study, there were eight intraparenchymal and one endobronchial hamartoma. The size of these parenchymal lesions ranges from 1 to $8 \mathrm{~cm}$; the maximum reported size was $25.5 \times 17.5 \times$ $6.5 \mathrm{~cm}$ with a weight of $1,134 \mathrm{~g} \cdot{ }^{13}$ The average size of these lesions in our study was $1 \mathrm{~cm}$. On histopathology, pulmonary hamartomas have various components like fat, cartilage, fibrous tissue, epithelium, and rarely bone marrow. Hamartomas may be further classified as lipomatous or chondromatous, depending on the dominant component. ${ }^{2,14}$ Sometimes, bronchial chondroma may be confused with endobronchial hamartoma. However, the absence of fat and mesenchymal stroma distinguishes a bronchial chondroma from hamartoma. ${ }^{15}$

Pulmonary hamartomas are benign neoplasms arising in the connective tissue beneath the walls of small bronchi. The clefts and epithelium lined spaces are believed to arise by the inclusion of the surface epithelium between the lobules of expanding connective tissue and cartilage., ${ }^{2,3}$

There have been rare reports of adenocarcinoma, squamous cell carcinoma or sarcoma arising from 
pulmonary hamartoma. ${ }^{16,17}$ No nuclear atypia, necrosis, increased mitotic activity or sarcomatoid change was observed in any of our cases. Some studies have suggested increased incidence of malignancy in cases with hamartomas. However, no pathophysiologic link could be established between pulmonary hamartoma and development of lung carcinoma. This can be explained by the fact that investigation for a malignancy in lung may lead to incidental detection of an asymptomatic hamartoma. ${ }^{15,17,18}$ There has been a recent report from our institute where a solitary pulmonary nodule, suspected to be metastatic deposit or a synchronous lung cancer, in a patient with a known primary malignancy, turned out to be pulmonary hamartoma on cytological examination. ${ }^{8}$ This very same case is also included in our case series to study the spectrum of cytological findings in detail. The two autopsy cases of our study were also known cases of different malignancies (one being small cell carcinoma of lung and the other being temporo-parietal astrocytoma) and were incidentally found to have solitary lung nodules which were proven to be hamartomas on microscopy.

The treatment for pulmonary hamartoma may range from enucleation, wedge resection to lobectomy, or rarely pneumonectomy; depending on size, location and growth pattern of the hamartoma, and presence or absence of symptoms and permanent parenchymal changes. $^{7}$

\section{CONCLUSION}

\section{Every solitary pulmonary nodule is not malignant}

It is important to correctly diagnose pulmonary hamartoma, a rare, yet benign neoplasm presenting as a solitary lung nodule and distinguish it from malignancy. Even an asymptomatic solitary pulmonary nodule, in a case with a known malignancy, may not always be malignant and may turn out to be a benign pulmonary hamartoma. It is important for the pathologists to keep the cytological and histopathological features of pulmonary hamartoma in mind in cases presenting as solitary pulmonary nodule, in order to reach the correct diagnosis and distinguish this rare yet benign primary tumour from malignant nodules.

\section{REFERENCES}

1. Albrecht E. Uber hamartome. Verh Dtsch Ges Pathol 1904;7:153157.

2. Bateson EM. So called hamartoma of the lung a true neoplasm of fibrous connective tissue of the bronchi. Cancer 1973; 31:14581467.

3. Cosio BG, Villena V, Echave-Sustaeta J, de Miguel E, Alfaro J, Hernandez L, et al. Endobronchialhamartoma. Chest 2002; 122:202-205.

4. Otani $Y$, Yoshida I, Kawashima O, Yamagishi T, Ishikawa S, Ohtaki A, et al. Benign tumors of the lung: A 20-year surgical experience. Surg Today 1997; 27:310-312.

5. Wiatrowska BA, Yazdi HM, Matzinger FR and MacDonald LL. Fine needle aspiration biopsy of pulmonary hamartomas. Radiologic, cytologic and immunocytochemical study of 15 cases. Acta Cytol 1995; 39:1167-1174.

6. Whyte RI and Donington JS. Hamartomas of the lung. Semin Thorac Cardiovasc Surg 2003; 15:301-304.

7. Pollock $A B, A l$ Hasan M, Roy TM and Byrd RP. Pulmonary hamartoma- an algorithmic approach to the diagnosis and management. Clin Pulm Med 2008;15:35-39.

8. Jindal A, Madan K, Nijhawan R and Singh N. Incidental pathologically proven pulmonary hamartoma in a patient with carcinoma tongue. BMJ Case Rep 2013. doi: 10.1136/bcr-2013008942.

9. Wood B, Swarbrick N and Frost F. Diagnosis of pulmonary hamartoma by fine needle biopsy. Acta Cytol.2008; 52:412-417.

10. Hughes JH, Young NA, Wilbur DC, Renshaw AA and Mody DR. Fine-needle aspiration of pulmonary hamartoma: a common source of false-positive diagnoses in the College of American Pathologists Inter laboratory Comparison Program in Nongynecologic Cytology. Arch Pathol Lab Med 2005;129:19-22.

11. Zakharov $V$ and Schinstine $M$. Hamartoma of the lung. Diagnostic Cytopathol 2008; 36:331-332.

12. Gjevre JA, Myers JL and Prakash UB. Pulmonaryhamartomas. Mayo Clin Proc. 1996; 71:14-20.

13. Ganti $S$, Milton R, Davidson $L$ and Anikin V. Giant pulmonary hamartoma. J Cardiothorac Surg 2006; 1:19.

14. Tomashefski JF. Benign endobronchial Mesenchymal tumors: their relationship to parenchymal pulmonary hamartomas. Am J Surg Pathol 1982; 6:531-540.

15. Jacob S, Mohapatra D and Verghese M. Massive chondroid hamartoma of the lung clinically masquerading as bronchogenic carcinoma. Indian J Pathol Microbiol 2008; 51:61-62.

16. Lee BJ, Kim HR, Cheon GJ, Koh JS, Kim CH and Lee JC. Squamous cell carcinoma arising from pulmonary hamartoma. Clin Nucl Med 2011; 36:130-131.

17. Ribet $M$, Jaillard-Thery $S$ and Nuttens MC. Pulmonary hamartoma and malignancy. J Thorac Cardiovasc Surg. 1994; 107:611-614.

18. Higashita R, Ichikawa $S$, Ban $T$, Suda $Y$, Hayashi $K$ and Takeuchi Y. Coexistence of lung cancer and hamartoma. Jpn J Thorac Cardiovasc Surg. 2001; 49:258-260. 